\title{
Non-familial degenerative disease and atrophy of brainstem and cerebellum
}

\section{Clinical and CT data in 47 patients}

\author{
Arthur Staal $^{1}$, Jan D. Meerwaldt ${ }^{1, *}$, Krijn J. van Dongen ${ }^{2}$, \\ Paul G.H. Mulder ${ }^{3}$ and Herman F.M. Busch ${ }^{1}$ \\ ${ }^{1}$ Department of Neurology, Erasmus University Rotterdam, ${ }^{2}$ Department of Radiology, St. Franciscus Hospital \\ Rotterdam, and ${ }^{3}$ Department of Biostatistics, Erasmus University Rotterdam, Rotterdam (The Netherlands)
}

(Received 21 August, 1989)

(Revised, received 9 October, 1989)

(Accepted 16 October, 1989)

SUMMARY

We studied the clinical features of 47 patients with a non-hereditary degenerative disease and with atrophy of brainstem or cerebellum or both in CT scanning. There was no relation between the CT findings and duration or severity of the disease, nor with the kind of the neurological signs which comprised ataxia, a hypokinetic rigid syndrome, oculomotor abnormalities, upper and lower motor neuron signs, orthostatic hypotension and dementia. The 2 main diagnoses were olivopontocerebellar atrophy (OPCA), or a combination of OPCA and striatonigral degeneration (SND). The differential diagnosis with Parkinson's disease and progressive supranuclear palsy was discussed. We concluded, that a CT scan is warranted in all cases of suspected Parkinson's disease, especially in those without tremor, and in cases of motoneuron disease with broad-based gait. In our patients with mainly hypokinesia and rigidity, levodopa treatment had no or brief beneficial effects. If ataxia predominated, OPCA appeared the most sensible diagnosis; if a hypokinetic-rigid syndrome predominated, the diagnoses SND plus OPCA appeared the most suitable. We assessed the degree of atrophy on CT subjectively, because an interobserver study of 60 normal CT scans, did not produce reliable measurements.

* We deeply regret that shortly before submission of this paper Dr. J.D. Meerwaldt passed away. Correspondence to: Prof. Dr. A. Staal, Department of Neurology, University Hospital Dijkzigt, Dr. Molewaterplein 40, 3015 GD Rotterdam, The Netherlands. 
Key words: Multiple system atrophy; Olivopontocerebellar atrophy; Cerebellar atrophy; Striatonigral degeneration; Shy-Drager disease

\section{INTRODUCTION}

Duvoisin and Plaitakis (1984) have stated that "the name olivopontocerebellar atrophy (OPCA) does not fully cover the pathological abnormalities", and that OPCA represents "the keyword for an admittedly heterogeneous but nevertheless distinctive clinicopathological entity" (Duvoisin 1984). In contrast, Harding (1984) argues that "it seems illogical to diagnose OPCA during life, since the clinical diagnosis is frequently not substantiated pathologically, and if so, the abnormalities are often more widely distributed than the name OPCA implies". Consequently, one should make "the same diagnosis in a parkinsonian patient with cerebellar atrophy on CT, as in another with late onset cerebellar ataxia, dementia and optic atrophy" (Harding 1987). Unpractical though this may seem, the same reasoning applies to other disorders with an impressive clinical variability, such as mitochondrial disorder. Furthermore, it may be very difficult to think of any degenerative neurological disease in which the name fully covers all its neuropathological abnormalities. Until more insight into the basic metabolic or enzymatic abnormality is provided, classification of degenerative disease remains unsatisfactory.

This controversy prompted us to present a consecutive series of 47 adult patients studied between 1980 and 1987. They suffered from a non-hereditary, progressive disease with a rather diverse symptomatology, comprising various combinations of cerebellar ataxia, hypokinetic rigid syndrome (HRS), oculomotor abnormalities, upper or lower motor neuron signs, and dementia.

Under suspicion of having multiple system atrophy (MSA) these patients were subjected to $\mathrm{CT}$ scanning. Patients with unequivocal cerebellar, and/or brainstem atrophy were included in the study. In order to distinguish from diffuse cerebral atrophy due to old age, we excluded cases with supratentorial atrophy, with the exception of isolated enlargement of the third ventricle. Although only two autopsies were performed, we are still confident to have reliably ruled out disorders other than degenerative disease. The autopsy diagnoses were OPCA in one, and OPCA plus striatonigral degeneration (SND) in the other.

In this study we aim to demonstrate that, following exclusion of non-degenerative neurological diseases by appropriate laboratory tests, CT scanning may establish a "definite diagnosis" of MSA, which denotes OPCA or OPCA plus SND. To our knowledge, this is the largest group of patients with non-hereditary atrophy of brainstem and/or cerebellum ever published. 


\section{PATIENTS AND METHODS}

\section{Clinical and laboratory diagnosis}

This study included 28 male and 19 female patients, with normal motor and mental development. Their age at the time of investigation ranged from 32 to 82 years (mean 60.4). The patients were seen independently by 2 of us (J. M. and A. S.) and there hardly was any disagreement concerning the clinical findings. All patients suffered from an idiopathic and progressive neurological disorder. Autonomic functions were not tested systematically, but autonomic symptoms and signs were noted in one case. Because the diverse symptomatology led to a clinical suspicion of MSA, we ordered CT scans in all but 1 (No. 33) of the patients and used subtentorial atrophy as an entrance criterium for the study.

Cerebral trauma, alcoholism, epilepsy and anticonvulsive medication, undernourishment, diabetes, thyroid, kidney and liver disease, and syphilis were prohibitive for entering the study. Further laboratory studies included normal hematology, urinanalysis and blood chemistry, and in thirteen patients normal findings in spinal fluid. Heredity was made unlikely by a meticulous family history, and by the fact that several patients belonged to large normal sibships. There was no known consanguinity of the patients' parents. However, a very rare spontaneous mutation for dominant heredity or a rare recessive case in a smaller sibship remains a possibility; but OPCA, if occurring in families, is nearly always autosomal dominant. Malignant disease was clinically unlikely, but has not been excluded by invasive radiological techniques. However, the long duration of the disease in the great majority of the patients, ranging from 6 months to 31 years with a mean duration of 4.3 years, made paraneoplastic disease improbable. Finally, the ever growing list of rare metabolic diseases was not extensively checked, but in adults these affections are extremely uncommon. To exclude this possibility, children and adolescents were not admitted into the study.

\section{$C T$ scanning}

The CT scans in the patients were made according to a standard protocol in 6 patients with an EMI CT 1010 machine, and in 40 patients with a Philips Tomoscan 350. Slices were made parallel to the orbitomeatal line (OML), determined on an enlarged lateral "scout view". The whole posterior fossa was covered (in 4-mm slices with the EMI scan and in 3-mm slices with the Philips scan) from the foramen magnum through the mesencephalon, including a large part of the third ventricle. Acquired data were postprocessed by reconstructive zoom with a field of view of 125-150 depending on head size. With standard window settings hard copies were made using a matrix camera. Of the supratentorial structures standard slices of $9 \mathrm{~mm}$ were made. Intravenous contrast was not administered.

In trying to get objective data in adults about the dimensions of different cerebral structures, we determined the interobserver agreement between 3 neurologists and 1 radiologist of our staff. Fifteen parameters (Huang and Plaitakis 1984) were measured (see Fig. 1) in 60 normal CT scans, which were drawn at random from our archives (Philips Tomoscan 350, using 9-mm slices parallel to a line through the lateral orbital 


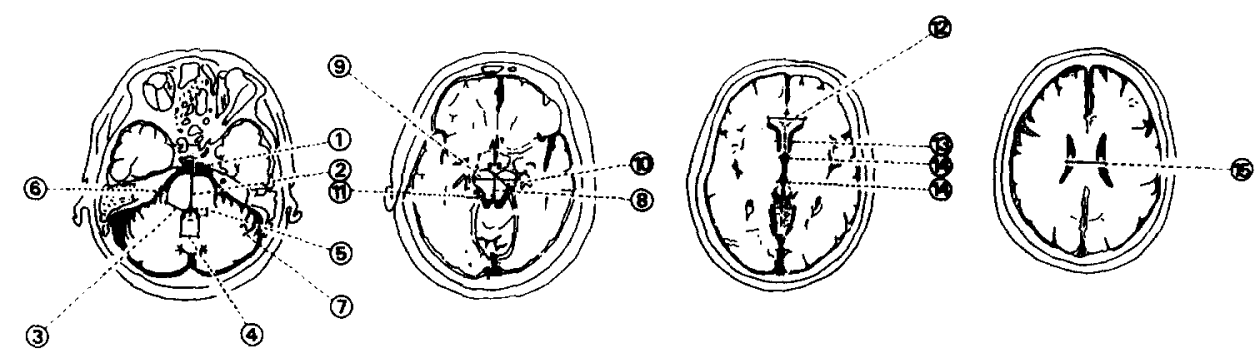

Fig. 1. prepontine cistern; 2: anteroposterior diameter of the pons; 3 : width of the brachium pontis; 4 : transverse diameter of the IVth ventricle; 5 : anteroposterior diameter of the IVth ventricle; $6:$ width of the cerebellopontine angle; 7: prebiventral sulcus; 8: anteroposterior diameter of the mesencephalon; 9 : transverse diameter of the mesencephalon; 10: transverse diameter of the basis pedunculi; 11: transverse diameter of the quadrigeminal cistern; 12: bifrontal cornual distance; 13: intercaudate distance; 14: transverse width of anterior and posterior part of the IIIrd ventricle; 15: biparietal ventricular distance.

corner and the external auditory meatus). All measures were taken with a specially designed ruler to compensate for the enlargement factor introduced by the field of view used.

In most of these normal CT scans we were unable to measure these parameters, especially in the infratentorial region but to a lesser extent also in the supratentorial region, as a result of patient positioning artifacts or of artifacts and the partial volume effect. In the measurements that could be taken, an analysis of variance was used in trying to calculate the interobserver agreement. We divided the total variance into an explainable part, namely patient and interobserver variance, and into an unexplained part, the so called residual variance. Because this residual variance formed the greatest part of the total variance, we could not calculate an interobserver agreement. The number of patients in which a variable could be measured, fluctuated from 0 to 19 (mean 8). Thus, due to the lack of normal data, we decided that three of the authors (J.M., A.S. and K.J.v.D.) should report independently their subjective estimate of hindbrain atrophy as,,++++++ and ++++ . Cerebellar atrophy was considered present only if individual folia were visible. It appeared that these subjective judgments led only to minor disagreement between the three authors, and these were easily settled in mutual consultation. Huang and Plaitakis (1984) did give exact measures of the atrophy found on CT in OPCA versus normal and age matched controls. The value of their data seems a bit questionable, however. There was a great variation in the positioning of patients and controls, namely between the superior and inferior orbitomeatal planes. Due to this factor the diameter of several posterior fossa structures may increase or decrease. Thereafter these differences were further accentuated by subtracting $5-10 \%$ from the values obtained in anteroposterior direction. Moreover they did no interobserver study.

\section{RESULTS}

\section{Clinical findings (Table 1)}

A mainly cerebellar syndrome was seen in only 7 patients (cases $1-7$ ) three of whom also had saccadic eye movements and/or vertical gaze paralysis. In 9 cases we 
noted cerebellar ataxia together with hyperreflexia and extensor plantar responses (cases 8-16). In 4 patients (cases 17-20) we found cerebellar ataxia with areflexia, once with fasciculations and signs of denervation on EMG, and twice with extensor plantar responses.

A hypokinetic rigid syndrome (HRS) was present in 27 patients (cases 21-47). In 7 of these (cases 21-27) the "pure" HRS superficially suggested idiopathic Parkinson's disease, but it was conspicuously without tremor. One patient (case 27) also had symptoms and signs of autonomic dysfunction. One patient (case 28) showed HRS with thenar and hypothenar atrophy. Five patients (cases 29-33) exhibited HRS in combination with cerebellar ataxia. In 4 patients (cases 34-37) we found HRS with hyperreflexia, extensor plantar responses and a spastic gait, but without pyramidal weakness. One of these also had peripheral motor neuron signs. In 1 patient we observed a HRS with cerebellar and pyramidal signs (case 38). Six patients (cases 39-44) showed HRS with dementia, in 2 of them also atrophy of the interosseus muscles, a third with fasciculations and in 3 others together with cerebellar ataxia. Finally, in 3 of these 27 patients (cases 45-47) we found vertical gaze paralysis, suggestive of progressive supranuclear palsy (PSP).

Many of the 27 patients with "pure" HRS and with HRS and other signs, deteriorated unusually rapidly: within 1-2 years, 17 of them were dependent in their daily life activities (Hoehn and Yahr grade III-V). Dopamine substitution therapy had no effect at all in 15 of the 27 patients with mainly HRS; 4 patients got an on/off syndrome within 2 years of treatment, 4 became rapidly psychotic under dopamine substitution, and in 4 no dopamine substitution was tried.

Abnormalities in ocular motor function were seen in 20 patients. Gaze induced nystagmus was found in 2 patients with HRS and once in a patient with HRS plus cerebellar ataxia. In 1 case with a mainly cerebellar syndrome a downbeat nystagmus was present. Optokinetic nystagmus (OKN) was absent in 6 patients: 3 times in patients with HRS, twice in mainly cerebellar cases, and once in a patient with cerebellar and pyramidal signs. Saccadic eye movements in combination with absent $\mathrm{OKN}$ were seen twice in patients with HRS, once in an otherwise mainly cerebellar case, and once in a patient with cerebellar and pyramidal signs. External ophthalmoplegia was only found once in a patient with HRS in combination with cerebellar ataxia (case 31). Paralysis of upward vertical gaze was present in 5 patients: in the 3 with a PSP-like syndrome (cases 45-47), and twice in mainly cerebellar syndromes (cases 6 and 7).

\section{CT findings (Table 2)}

As stated before, the conditions for being included in this study were clinical suspicion of idiopathic MSA and atrophy on CT, in different degrees and combinations, of pons, mesencephalon or cerebellum, with widening of the surrounding cysterns.

In relation to the clinical findings, we made the following observations:

In 5 of the 7 patients with a mainly cerebellar syndrome, cerebellar folia were visible and in 6 there existed an enlarged cerebellopontine angle. Five of these patients with a mainly cerebellar syndrome had atrophy of the mesencephalon as well. Of the 30 patients, who among other signs also had cerebellar ataxia, 8 had no atrophy of the 


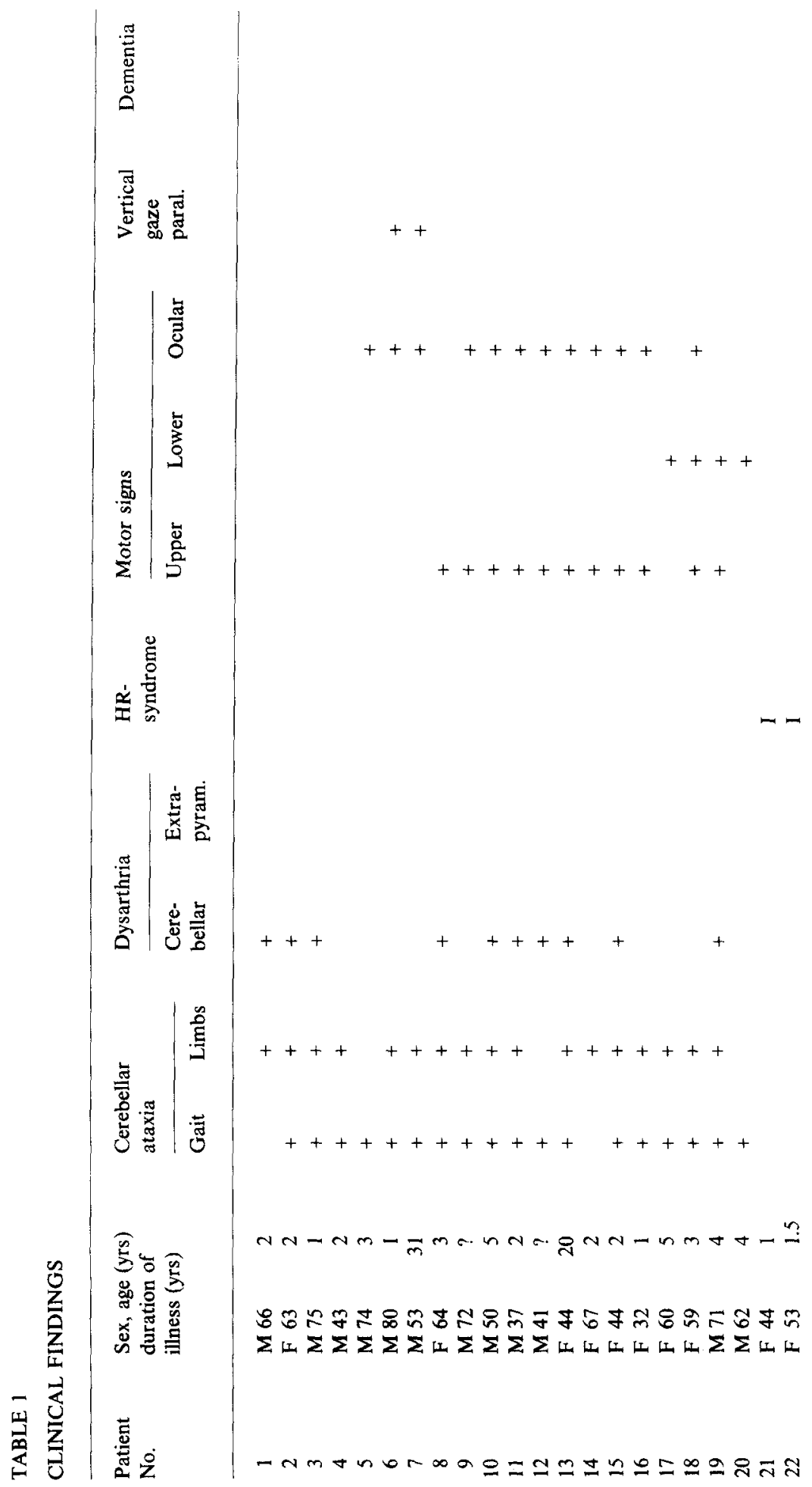




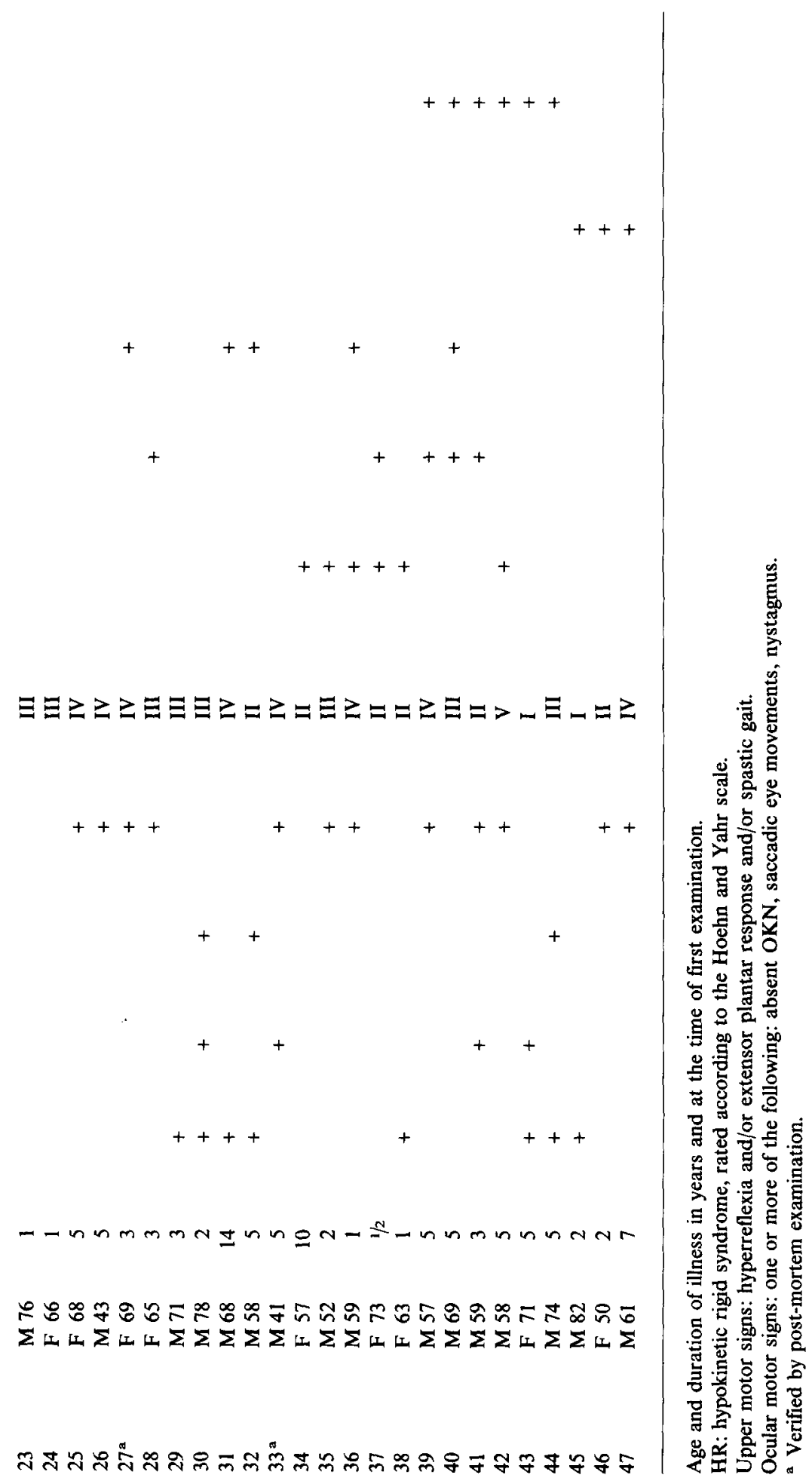


TABLE 2

FINDINGS ON CT SCANNING

\begin{tabular}{|c|c|c|c|c|c|c|c|}
\hline \multirow{2}{*}{$\begin{array}{l}\text { Patient } \\
\text { No. }\end{array}$} & \multicolumn{3}{|c|}{ Atrophy } & \multicolumn{4}{|c|}{ Enlargement } \\
\hline & Pons & Midbrain & $\begin{array}{l}\text { Cere- } \\
\text { bellum }\end{array}$ & $\begin{array}{l}\text { IVth } \\
\text { ventricle }\end{array}$ & $\begin{array}{l}\text { Cereb.- } \\
\text { pontine } \\
\text { angle }\end{array}$ & $\begin{array}{l}\text { Cisterna } \\
\text { ambiens }\end{array}$ & $\begin{array}{l}\text { IIIrd } \\
\text { ventricle }\end{array}$ \\
\hline 1 & & + & + & & ++ & $+t$ & + \\
\hline 2 & & + & ++ & + & & + & \\
\hline 3 & & ++ & + & & + & + & \\
\hline 4 & & & +++ & ++ & ++ & & \\
\hline 5 & ++ & ++ & & & +++ & & ++ \\
\hline 6 & ++ & + & & & +++ & & + \\
\hline 7 & & & ++ & ++ & $++t$ & & \\
\hline 8 & ++ & ++ & ++ & $+t$ & + & + & \\
\hline 9 & ++ & & & ++ & ++ & & \\
\hline 10 & & + & ++ & + & & ++ & \\
\hline 11 & & $+t$ & ++ & $+t$ & ++ & + & \\
\hline 12 & & & + & + & ++ & & \\
\hline 13 & ++ & ++ & +++ & ++ & & & \\
\hline 14 & & ++ & + & + & & & \\
\hline 15 & & + & + & + & & & \\
\hline 16 & ++ & ++ & ++ & +++ & + & ++ & \\
\hline 17 & & & + & & & & \\
\hline 18 & & + & & + & & + & \\
\hline 19 & ++ & + & + & & ++ & & \\
\hline 20 & +++ & +++ & ++ & ++ & +++ & ++ & \\
\hline 21 & +++ & ++ & ++ & +++ & ++ & ++ & \\
\hline 22 & & + & + & & ++ & ++ & + \\
\hline 23 & & ++ & & & + & + & \\
\hline 24 & ++ & & + & +++ & & + & \\
\hline 25 & & + & ++ & & + & + & \\
\hline 26 & & ++ & ++ & & ++ & ++ & \\
\hline 27 & & + & + & ++ & + & & \\
\hline 28 & & + & + & & + & & \\
\hline 29 & ++ & + & +++ & $+t$ & +++ & & \\
\hline 30 & ++ & + & +++ & ++ & +++ & & \\
\hline 31 & & $+t$ & & & ++ & + & + \\
\hline 32 & & ++ & ++ & & ++ & ++ & \\
\hline $33^{a}$ & & ++ & ++ & & & & \\
\hline 34 & + & & ++ & & ++ & & \\
\hline 35 & +++ & ++ & $++t+$ & + & ++++ & ++ & \\
\hline 36 & & + & ++ & & ++ & ++ & \\
\hline 37 & + & & & + & & + & + \\
\hline 38 & + & + & + & & & & + \\
\hline 39 & & & ++ & ++ & & ++ & \\
\hline 40 & ++ & & & & ++ & & \\
\hline 41 & & ++ & & & & ++ & \\
\hline 42 & & + & + & & ++ & & + \\
\hline 43 & ++ & ++ & & + & & + & + \\
\hline 44 & & ++ & & & & ++ & + \\
\hline 45 & ++ & & + & & ++ & & + \\
\hline 46 & + & ++ & + & + & & + & \\
\hline 47 & + & ++ & ++ & + & & ++ & \\
\hline
\end{tabular}

a From patient 33 no CT scan was available. The atrophy was assessed at autopsy. 
cerebellum. Of the 17 patients without ataxia an atrophic cerebellum was found in 14 . Of the 27 patients with mainly HRS only 7 had no cerebellar atrophy on CT. In 2 of the 3 patients with a clinical syndrome suggestive for PSP, atrophy was found of cerebellum, pons and mesencephalon; in the third patient the CT scan showed atrophy of the pons and the cerebellum. In 11 cases the third ventricle was also enlarged.

\section{DISCUSSION}

Our findings demonstrate a striking heterogeneity in our patients with clinical signs of degenerative disease and atrophy of the hindbrain on CT. If ataxia is the most important sign, one may choose the clinical denomination of OPCA. If a hypokinetic rigid syndrome dominates the clinical picture, one may diagnose a combination of SND and OPCA, as has been reported before (Adams et al. 1964; Gosset et al. 1983).

\section{Differential diagnosis with Shy-Drager disease}

If a patient with HRS, with or without ataxia, also has autonomic disturbances (case 27), one may consider the diagnosis of Shy-Drager disease. Clinical and neuropathological overlap between OPCA, SND and Shy-Drager disease has been stressed by Ropper (Scully et al. 1983).

\section{Differential diagnosis with idiopathic Parkinson's disease}

An important and practical conclusion from our study seems to be that these diseases may often mimic idiopathic Parkinson's disease, however, in our patients without tremor. In 27 of our 47 patients hypokinesia and rigidity were the most outstanding findings, and 17 of them exhibited HRS without any ataxia. In one of these, saccadic ocular pursuit movements were present. Without a CT scan this could easily have led to the wrong conclusion: Parkinson's disease. In these cases, at least in our hands, levodopa substitution therapy was either of no or only of very short benefit.

\section{Differential diagnosis with PSP}

Clinical differentiation between OPCA and PSP is notoriously difficult if at all possible (Duvoisin 1987). Thus, to us it seems remarkable that in the recent series of 34 patients with PSP of Maher and Lees (Maher and Lees 1986) nothing was said about the differential diagnosis with OPCA especially since no autopsies were reported. We found only 20 cases in which the gross anatomy and the clinical findings in PSP were reported in detail in neuropathologically proven cases (Behrman et al. 1969; Calzetti et al. 1987; Dix et al. 1971; Jellinger 1971; Steele et al. 1961; Steele 1972). It appeared that cases of pathologically proved PSP may have had broad-based gait, but never ataxia of the limbs. On autopsy gross atrophy of the brainstem was found in only 5 of these twenty cases, but gross atrophy of the cerebellum has not been described in PSP.

Perhaps this may lead to the conclusion that atrophy of the cerebellum on CT makes the diagnosis of PSP untenable. However, only atrophy of the brainstem on CT, without clear-cut cerebellar signs does not give a conclusion either way, unless peripheral 
motor signs point to OPCA. Reviewing our patients with these points in mind, there were 11 patients with only brainstem atrophy, but 6 of these had enlarged cerebellopontine angles, which has not been described in PSP. Thus, there remain 5 cases without atrophy of the cerebellar folia and with normal cerebellopontine angles (cases 18, 37, 41,43 and 44). All these had clear-cut cerebellar signs and/or peripheral motor neuron signs, and none had vertical gaze paralysis.

Finally, 3 of our patients (cases 45-47) who were described as having a PSP-like syndrome only had a vertical upward gaze paralysis.

Thus we feel reasonably certain that none of our 47 patients had PSP.

\section{Differential diagnosis with amyotrophic lateral sclerosis}

In case 20 originally the diagnosis of motor neuron disease was made, because the slightly broad-based gait was not taken into account. After a few years, when cerebellar dysarthria became evident, a CT scan disclosed pronounced cerebellar and brainstem atrophy.

\section{Relation between clinical signs and CT findings}

Atrophy of pons, cerebellum and mesencephalon was not related with the clinical picture. In patients with HRS we found cerebellar atrophy as often as in ataxic patients. There was no relationship whatever between the duration of the disease and the severity of the atrophy on CT. We observed no abnormalities other than infratentorial atrophy, especially no infarcts nor periventricular leucoencephalopathy.

In conclusion, a CT scan may change the diagnosis of suspected idiopathic Parkinson's disease, especially if there is no tremor. A CT scan should also be made if PSP is suspected, and in motor neuron disease when gait is slightly broad-based.

The patient's disease may then appear to belong to a multiple system degeneration of which the atrophy of brainstem and cerebellum as found on CT may be an important part of the gross pathology. We suggest that a clinical diagnosis of OPCA or a combination of OPCA and SND may then be the most probable one. If, and how often CT scans in OPCA may be normal while microscopically OPCA is diagnosed, is unknown.

\section{ACKNOWLEDGEMENTS}

This is to thank K. Ronday, W. Koops and E.F.M. Wijdicks, M.D. for their cooperation in the interobserver study, Prof. S. Stefanko, M.D. for his neuropathological examination of cases 25 and 32 and Mrs. E.M. Vermeulen-Yark for extensive secretarial work. We gratefully acknowledge Prof. H. van Crevel, M.D., who critically read the manuscript.

\section{REFERENCES}

Adams, R. D., L. van Bogaert and H. VanderEecken (1964) Striato-nigral degeneration. J. Neuropathol. Exp. Neurol., 23: 584-608. 
Behrman, S., J. D. Caroll, I. Janota et al. (1969) Progressive supranuclear palsy. Clinico-pathological study of four cases. Brain, 92: 663-678.

Calzetti, S., F. Gemignani, A. Lechi et al. (1987) Progressive supranuclear palsy in the course of subclavian steal syndrome. Acta Neuropathol. (Berlin), 7 (Suppl.): 372-374.

Dix, M.R., M.J.G. Harrison and P.D. Lewis (1971) Progressive supranuclear palsy. A report of 9 cases with particular reference to the mechanism of the oculomotor disorder. J. Neurol. Sci., 13: 237-256.

Duvoisin, R.C. (1984) An apology and an introduction to the olivopontocerebellar atrophies. In: Duvoisin R. C. and A. Plaitakis (Eds.), Advances in Neurology, The Olivopontocerebellar Atrophies, Raven Press, New York, pp. 5-12.

Duvoisin, R.C. (1987) The olivopontocerebellar atrophies. In: Marsden, C. D. and S. Fahn (Eds.), Movement Disorders, 2, Butterworths, London, pp. 249-269.

Gosset, A., J. F. Pellissier, F. Delpuech et al. (1983) Dégénerescence striato-nigrique associée à une atrophie olivo-ponto-cérébelleuse. Rev. Neurol. (Paris), 139: 125-139.

Harding, A.E. (1984) The Hereditary Ataxias and Related Disorders. Churchill Livingstone, Edinburgh, pp. 10.

Harding, A. E. (1987) Olivopontocerebellar atrophy is not a useful concept. In: Marsden, C. D. and S. Fahn (Eds.), Movement Disorders 2, Butterworths, London, pp. 269-271.

Huang, Y.P. and A. Plaitakis (1984) Morphological changes of olivopontocerebellar atrophy in computed tomography and comments on its pathogenesis. In: Duvoisin, R.C. and A. Plaitakis (Eds.), Advances in Neurology, The Olivopontocerebellar Atrophies, Raven Press, New York, pp. 39-85.

Jellinger, K. (1971) Progressive supranuclear palsy (subcortical argyrophilic dystrophy). Acta Neuropathol. (Berl.), 19: 347-352.

Maher, E.R. and A.J. Lees (1986) The clinical features and natural history of the Steele-RichardsonOlszewski syndrome (progressive supranuclear palsy). Neurology, 36: 1005-1008.

Scully, R.E., E.J. Mark and B.U. McNeely (Eds.), (1983) Case records of the Massachusetts General Hospital. Case 23-1983. N. Engl. J. Med., 308: 1406-1414.

Steele, J.C. (1972) Progressive supranuclear palsy. Brain, 95: 693-704.

Steele, J.C., J.C. Richardson and J. Olszewski (1961) Progressive supranuclear palsy. Arch. Neurol., 10: 333-359. 